\title{
Critical Casimir forces in the presence of random surface fields
}

\author{
A. Maciołek, ${ }^{1,2,3, *}$ O. Vasilyev, ${ }^{1,2}$ V. Dotsenko, ${ }^{4,5}$ and S. Dietrich ${ }^{1,2}$ \\ ${ }^{1}$ Max-Planck-Institut für Intelligente Systeme, \\ Heisenbergstr. 3, D-70569 Stuttgart, Germany \\ ${ }^{2} I V$. Institut für Theoretische Physik, Universität Stuttgart, \\ Pfaffenwaldring 57, D-70569 Stuttgart, Germany \\ ${ }^{3}$ Institute of Physical Chemistry, Polish Academy of Sciences, \\ Kasprzaka 44/52, PL-01-224 Warsaw, Poland \\ ${ }^{4}$ LPTMC, Universite Paris VI - 75252 Paris, France \\ ${ }^{5}$ L.D. Landau Institute for Theoretical Physics, 119334 Moscow, Russia
}

(Dated: October 12, 2018)

\begin{abstract}
We study critical Casimir forces $(\mathrm{CCF}) f_{\mathrm{C}}$ for films of thickness $L$ which in the three-dimensional bulk belong to the Ising universality class and which are exposed to random surface fields (RSF) on both surfaces. We consider the case that, in the absence of RSF, the surfaces of the film belong to the surface universality class of the so-called ordinary transition. We carry out a finite-size scaling analysis and show that for weak disorder CCF still exhibit scaling, acquiring a random field scaling variable $w$ which is zero for pure systems. We confirm these analytic predictions by MC simulations. Moreover, our MC data show that $f_{\mathrm{C}}$ varies as $f_{\mathrm{C}}(w \rightarrow 0)-f_{\mathrm{C}}(w=0) \sim w^{2}$. Asymptotically, for large $L, w$ scales as $w \sim L^{-0.26} \rightarrow 0$ indicating that this type of disorder is an irrelevant perturbation of the ordinary surface universality class. However, for thin films such that $w \simeq 1$, we find that the presence of RSF with vanishing mean value increases significantly the strength of $\mathrm{CCF}$, as compared to systems without them, and shifts the extremum of the scaling function of $f_{\mathrm{C}}$ towards lower temperatures. But $f_{\mathrm{C}}$ remains attractive.
\end{abstract}

\footnotetext{
* maciolek@is.mpg.de corresponding author
} 


\section{INTRODUCTION}

Critical Casimir forces $(\mathrm{CCF})$ arise between surfaces confining a fluid which is brought thermodynamically close to its bulk critical point [1]. There they are described by universal scaling functions which are determined by the universality class of the bulk liquid and the surface universality classes of the confining surfaces [2]. Interfaces confining ${ }^{4} \mathrm{He}$ near its superfluid transition belong to the surface universality class of the so-called ordinary transition corresponding to Dirichlet boundary conditions (BC) for the superfluid order parameter [3]. Surfaces confining classical binary liquid mixtures near their demixing transition belong to the universality class of the so-called normal transition [4 8], which is characterized by a strong effective surface field acting on the deviation of the concentration from its critical value serving as the order parameter. The surface field describes the preference of the container wall for one of the two species forming the binary liquid mixture. For ${ }^{3} \mathrm{He} /{ }^{4} \mathrm{He}$ mixtures near their tricritical point both types of BC can occur [9]. These experimental findings agree with corresponding theoretical analysis [10 13] and Monte Carlo simulations [14 16] of suitable model systems representing the aforementioned universality classes and the crossover between them [17-20]. Across the various universality classes the magnitude, the shape, and the sign of the universal scaling functions of the CCF vary strongly. For example, for films with ordinary-ordinary $(o, o)$ or normal-normal $(+,+) \mathrm{BC}$ at the two surfaces CCF are attractive CCF whereas for opposing $(+,-)$ BC they are repulsive.

The sign of the surface fields depends on the chemical composition of the wall surfaces. They can be designed by suitable surface treatments which, e.g., render hydrophilic or hydrophobic surfaces [6, 7]. In the context of CCF spatially varying surface compositions have been studied experimentally for a smooth lateral gradient [21] and for well defined alternating stripes [22]. Without dedicated preparation efforts, the surfaces typically carry random chemical heterogeneities due to adsorbed impurities which act as local surface fields. If kinetically frozen they form quenched disorder. Quenched random-charge disorder on surfaces of dielectric parallel walls at a distance $L$ leads to long-ranged forces $\sim L^{-2}$ even if they are net neutral [23, 24], which dominates the pure van der Waals term $\sim L^{-3}$.

Here we study CCF emerging under the influence of randomly quenched surface fields. Specifically, we consider the Ising bulk universality class and a situation in which the mean value of the surface fields vanishes. As a rough guideline this addresses systems in which 
droplets of the demixed binary liquid mixture form a contact angle of $90^{\circ}$ with the chemically disordered substrate (see the intermediate substrate compositions in Ref. [21]). We analyze slabs of thicknesses $L$. In the corresponding limit $L \rightarrow \infty$, leading to two semi-infinite systems, the influence of random surface fields has been studied in the context of wetting (for reviews see Ref. [25]) and surface critical phenomena [26 28] (for a review see Ref. [29]). In particular, the Harris criterion concerning the relevance of disorder for bulk critical phenomena has been generalized to surface critical behavior [27]. Within the framework and limitations of a weak-disorder expansion, quenched random surface fields with vanishing mean value are expected to be irrelevant if the pure system belongs to the ordinary surface universality class [27]. For the three-dimensional $(d=3)$ Ising model in Ref. [26] this was pointed out and confirmed by Monte Carlo simulations.

Parallel to the present study, in Ref. [30] the case of random surface fields acting on only one of the two confining surfaces, with the other surface belonging to the universality class of the normal transition, has been analyzed for the 'improved' Blume-Capel model [16, 31, 32]. The scaling functions of CCF in that system have been obtained by using Monte Carlo simulations and finite-size scaling. We note that for complex fluids disorder effects on Casimir-like interactions can be dominant. This has been shown recently for nematic liquid-crystalline films bounded by two planar surfaces, one of which exhibiting a random distribution of the preferred anchoring axis in the so-called easy direction [33]. In this case of quenched disorder, the effects of disorder onto the fluctuation-induced interaction between the surfaces are dominant at intermediate film thicknesses.

Our presentation is organized as follows. In Sec. [I] we present a scaling analysis from which we derive a random field finite-size scaling variable. Section III is devoted to MC simulations. In Sec. IIIA we define our system and provide the details of our numerical method of determining the $\mathrm{CCF}$ and its scaling functions from the MC simulation data. Section IIIB contains our results. We provide a summary and conclusions in Sec. IV.

\section{RANDOM FIELD SCALING}

First, we consider pure systems. Within mean field theory, near the ordinary transition of semi-infinite systems the order parameter profile exhibits an extrapolation length $1 / c$; $c=\infty$ is the fixed point of the ordinary transition (o) corresponding to Dirichlet BC [2]. 
Close to this ordinary transition there is a single linear scaling field $g_{1}=H_{1} / \tilde{c}^{y_{c}}$ associated with the dimensionless surface field of strength $H_{1}$ and the surface enhancement parameter $\tilde{c}=c a$, where $a$ is a characteristic microscopic length scale of the system [2] such as the amplitude $\xi_{0}^{ \pm}$of the bulk correlation length $\xi_{b}\left(t=\frac{T-T_{c}}{T_{c}} \rightarrow 0^{ \pm}\right) \simeq \xi_{0}^{ \pm}|t|^{-\nu}$ (which stands for asymptotic equality). In the following all lengths, such as $L$ and $1 / c$, are expressed in units of $a$. The above scaling exponent is $y_{c}=\left(\Delta_{1}^{s p}-\Delta_{1}^{\text {ord }}\right) / \Phi$, where $\Delta_{1}^{\text {ord }}$ and $\Delta_{1}^{s p}$ are the surface counterparts at the ordinary and special transition, respectively, of the bulk gap exponent $\Delta$, and $\Phi$ is a crossover exponent [2]. Within mean field theory one has $y_{c}=1$ whereas $y_{c}(d=3) \approx 0.87$ [2, 19, 34]. Close to the critical point, the singular part $f_{\text {sing }}$ of the free energy per $k_{B} T$ and divided by $A L$ of a film of thickness $L$ and the surface area $A$ depends on three (dimensionless) scaling fields: $t$, the bulk ordering field $h_{b}$, and $g_{1}$; it depends on $L$ but not on $A$. For $L \gg a$, it is a generalized homogeneous function so that $f_{\text {sing }}\left(t, h_{b}, g_{1} ; L^{-1} ; a\right) \simeq b^{-d} f_{\text {sing }}\left(b^{y_{t}} t, b^{y_{b}} h_{b}, b^{y_{1}} g_{1} ; b L^{-1} ; a\right)$ for any dimensionless rescaling factor $b>0$ and bulk spatial dimension $d \geq 2$. The scaling exponents $y_{t}, y_{b}$, and $y_{1}$ are related to the aforementioned critical exponents: $y_{t}=1 / \nu, y_{b}=\Delta / \nu$, and $y_{1}=\Delta_{1}^{\text {ord }} / \nu$. (Note that $L^{-1}$ can be treated as a scaling field with scaling exponent equal to 1.) Setting $b=L / a$ one obtains (omitting the nonuniversal amplitudes of the scaling fields) $f_{\text {sing }}\left(t, h_{b}, g_{1} ; L^{-1} ; a\right) \simeq(L / a)^{-d} f_{\text {sing }}\left((L / a)^{1 / \nu} t,(L / a)^{\Delta / \nu} h_{b},(L / a)^{\Delta_{1}^{\text {ord }} / \nu} g_{1} ; a ; a\right)$.

We now consider a Gaussian distribution of surface fields with the ensemble averages $\overline{H_{1}(\mathbf{r})}=0$ and $\overline{H_{1}(\mathbf{r}) H_{1}\left(\mathbf{r}^{\prime}\right)}=h^{2} \delta\left(\mathbf{r}-\mathbf{r}^{\prime}\right) ; \mathbf{r}$ and $\mathbf{r}^{\prime}$ denote dimensionless lateral positions. In this case, the above finite-size scaling relation for the free energy density is modified. A heuristic renormalization group argument [26, 35] predicts that the scaling exponent of a random surface field is $y_{1}-(d-1) / 2$. This argument is based on the assumption that in a surface block of side length $b$ and area $b^{d-1}$ the effect, on the pure system, of small quenched local fluctuations of the surface field $H_{1}(\mathbf{r})$ of average magnitude $h$ and zero mean is the same as that of the average strength $\left(\overline{\left(H_{1}^{c g}(\mathbf{r})\right)^{2}}\right)^{1 / 2}$ of the coarse grained random field $H_{1}^{c g}=\sum_{i=1}^{\mathcal{N}_{b}} H_{1}\left(\mathbf{r}_{i}\right)$ uniformly distributed over the $\mathcal{N}_{b}$ sites $\mathbf{r}_{i}$ of that block. Since $H_{1}^{c g}$ is the sum of $\mathcal{N}_{b}$ uncorrelated random (surface) fields one has $\left(\overline{\left(H_{1}^{c g}(\mathbf{r})\right)^{2}}\right)^{1 / 2} \sim h \sqrt{\mathcal{N}_{b}}$. With $\mathcal{N}_{b} \sim b^{d-1}$ one obtains $\left(\overline{\left(H_{1}^{c g}(\mathbf{r})\right)^{2}}\right)^{1 / 2} / \mathcal{N}_{b} \sim h / \sqrt{\mathcal{N}_{b}} \sim h b^{-(d-1) / 2}$.

A real space renormalization-group transformation replaces such a block by a single site of 
the renormalized system with the associated quenched fluctuation of strength $b^{y_{1}} b^{-(d-1) / 2} h$. Thus in a system with random surface fields the appropriate scaling variable, which replaces $(L / a)^{\Delta_{1}^{\text {ord }} / \nu} g_{1}$ for the pure system, is

$$
w \equiv(L / a)^{\Delta_{1}^{o r d} / \nu-(d-1) / 2} h / c^{y_{c}}=(L / a)^{y_{1}-(d-1) / 2} \tilde{g}_{1},
$$

where $\tilde{g}_{1}=h / c^{y_{c}}$; in the following we choose $a=\xi_{0}^{+}$. As in our previous study [19], for the three-dimensional $(d=3)$ Ising model we take $\Delta_{1}^{\text {ord }} \approx 0.46(2)$ [34], $\Delta_{1}^{s p} \approx 1.05$ [2], $\Phi \approx 0.68$ [2], and $\nu \approx 0.63$ [16, 36], and obtain $y_{1}-(d-1) / 2 \approx-0.26(6)$. (More accurate estimates for the surface critical exponents at the special and ordinary transitions were obtained recently from MC simulations [37]. They yield $y_{c} \approx 1.282(5)$ and $y_{1} \approx 0.7249(6)$ so that $y_{1}-(d-1) / 2 \approx-0.2750(4)$. We have checked that using these latter estimates does not change the conclusion of our study and yields very similar results.) Within mean field theory, i.e., for $d=4$, one has $\Delta_{1}^{\text {ord }}=\nu=1 / 2[2]$ so that $y_{1}-(d-1) / 2=-1 / 2$. In the marginal case $d=2$ one has $y_{1}-(d-1) / 2=0$, due to $\nu=1$ and $\Delta_{1}^{\text {ord }}=1 / 2[2]$. Accordingly, for the $d=3$ Ising model one has $w=\left(h / c^{0.87}\right)\left(L / \xi_{0}^{+}\right)^{-0.26}$ whereas within mean field theory $w=(h / c)\left(L / \xi_{0}^{+}\right)^{-1 / 2}$.

At vanishing bulk ordering field $h_{b}=0$, the singular part of the excess free energy $f_{\text {sing }}^{e x}\left(L^{-1}\right)=f_{\text {sing }}\left(L^{-1}\right)-f_{\text {sing }}(0)$ (per $k_{B} T$ and divided by $A L$ ) satisfies (see Eq. (3.18) in Ref. [50] and Eqs. (1.7) and (1.8) in Ref. [48])

$$
\begin{aligned}
f_{\text {sing }}^{e x}\left(t, h_{b}=0, \tilde{g}_{1} ; L^{-1} ; \xi_{0}^{+}\right) & \simeq\left(L / \xi_{0}^{+}\right)^{-d} f_{\text {sing }}^{e x}\left(\left(L / \xi_{0}^{+}\right)^{1 / \nu} t,\left(L / \xi_{0}^{+}\right)^{y_{1}-(d-1) / 2} \tilde{g}_{1} ; \xi_{0}^{+}, \xi_{0}^{+}\right) \\
& \left.=L^{-d} \Theta\left(L / \xi_{0}^{+}\right)^{1 / \nu} t,\left(L / \xi_{0}^{+}\right)^{y_{1}-(d-1) / 2} \tilde{g}_{1}\right)
\end{aligned}
$$

Accordingly, the critical Casimir force per area $A$ and in units of $k_{B} T$ defined as

$$
f_{\mathrm{C}} \equiv-\frac{\partial\left(L f_{\text {sing }}^{e x}\right)}{\partial L}
$$

satisfies

$$
\left.f_{\mathrm{C}}(T, L, h) \simeq L^{-d} \vartheta\left(x=L / \xi_{0}^{+}\right)^{1 / \nu} t, w=\left(L / \xi_{0}^{+}\right)^{y_{1}-(d-1) / 2} \tilde{g}_{1}\right) .
$$

As follows from Eq. (3), the scaling function $\vartheta$ is related to $f_{\text {sing }}^{e x}$ and its derivatives. Because the scaling exponent of the random surface field is negative, the scaling field $\tilde{g}_{1}$ is irrelevant in the sense of renormalization-group theory. Under the assumption that the scaling function $\vartheta$ can be expanded in powers of the irrelevant field $\tilde{g}_{1}$, one obtains the critical Casimir force

$$
\begin{aligned}
f_{\mathrm{C}}(T, L, h) & \simeq L^{-d} \vartheta+\tilde{g}_{1}\left(\xi_{0}^{+}\right)^{y_{1}-(d-1) / 2} L^{-d-\left(y_{1}-(d-1) / 2\right)} \vartheta_{1} \\
& +\tilde{g}_{1}^{2}\left(\xi_{0}^{+}\right)^{2\left(y_{1}-(d-1) / 2\right)} L^{-d-2\left(y_{1}-(d-1) / 2\right)} \vartheta_{2}+\ldots,
\end{aligned}
$$


where $\vartheta_{1}$ is the first derivative of $\vartheta$ with respect to $w$ and $\vartheta_{2}$ is the second derivative. In Eq. (5) the function $\vartheta$ and its derivatives are evaluated at $w=0$. For sufficiently thick films the effect of disorder is expected to be negligible, i.e., the second variable in Eq. (4) can be neglected in the limit $L \rightarrow \infty$. However, in $d=3$ the exponent $y_{1}-(d-1) / 2$ is small and for thin films the corrections to scaling due to $\tilde{g}_{1}$ (i.e., the second and perhaps also the third term in the expansion in Eq. (5) ) can be large and hence important for experimental realizations. For large $\tilde{g}_{1}$ and thin films, it may happen that in Eq. (5) even more terms have to be included in order to capture the behavior of $f_{\mathrm{C}}(T, L, h)$.

In the pure case, there is a length $\ell_{\text {ord }}=g g_{1}^{-1 / y_{1}}$ associated with the scaling field $g_{1}$ [38], where $g$ is a nonuniversal amplitude, which can be small or large depending on the relative strength of $H_{1}$ and $\tilde{c}$. Upon approaching the ordinary transition, i.e., in the limit $c \rightarrow+\infty$ at fixed $H_{1}$ one has $\ell_{\text {ord }} \rightarrow \infty$. On the other hand, in the limit $H_{1} \rightarrow \infty$ at fixed $c>0$, one has $\ell_{\text {ord }} \rightarrow 0$ at the normal transition $(+)$. In $d=3$ one has $\ell_{\text {ord }}=g_{1}^{-1.3793(9)}$. Various studies of Ising systems in the film geometry [17-19, 39 43] showed that close to the ordinary transition the critical properties of the film of thickness $L$ are particularly sensitive to the strength of the surface fields, i.e., whether the length scale $\ell_{\text {ord }}$ becomes comparable to or even larger than $L$, where criticality means $L, \ell_{\text {ord }} \ll \xi_{b}$. In particular, in films with identical surface fields, the absolute value of the critical Casimir force at the bulk critical temperature (characterized by the critical Casimir amplitude) as a function of the surface field $H_{1}$ exhibits a minimum at $L \simeq \ell_{\text {ord }}$ [19, 41]. For equal surfaces, also the effect of the variation of the amplitude of $H_{1}$ on the temperature dependence of the critical Casimir force, i.e., the crossover behavior between the ordinary and the normal surface universality classes, has been studied [17, 19, 43]. For $L / \ell_{\text {ord }} \approx 1$ these results show strong deviations of the force scaling function from its universal fixed-point behavior such as the occurrence of two minima, one above and one below $T_{c}$, but no change in sign as the temperature is varied.

In the case of disorder, due to the scaling exponent $y_{1}-(d-1) / 2$ of a random surface field one can identify a length scale $\ell$ associated with the latter as $\ell=\kappa\left(h / c^{y_{c}}\right)^{-1 /\left(y_{1}-(d-1) / 2\right)}$ where $\kappa$ is a microscopic length. In $d=3$ one has $\ell=\kappa\left(h / c^{0.87}\right)^{3.85}$. Conversely to $\ell_{\text {ord }}$ in the pure case, at the ordinary transition, i.e., in the limit of $c \rightarrow+\infty$ at fixed $h$, $\ell$ vanishes. One can also consider the limit of large $h$ at fixed $c>0$ which, however, does not correspond to the normal transition. In the case of a random surface field, $h$ is a standard deviation of 
the Gaussian probability distribution of the surface fields. Upon increasing $h$ the probability distribution broadens so that also strong surface fields occur. Since the presence of a surface field of strength $\left|H_{1}\right| \gtrsim 1$ eliminates the fluctuations of the boundary Ising spins, we expect that for $h \gtrsim 1$ the actual value of $h$ does no longer matter and that accordingly the variation of the free energy with $h$ levels off. The typical microscopic configuration of a surface layer exposed to the realization of random surface fields with large standard deviation $h$ will be that of non-fluctuating spins distributed spatially almost at random. (Surface fields with $\left|H_{1}\right| \lesssim 1$ will give rise to some correlation effects.) For the finite size of a surface as used in numerical simulations, fluctuations about the zero mean value of the random surface field are expected to result in a nonvanishing, albeit small, surface magnetization. For thin films and for suitably chosen $h$ and $c$ such that $\kappa h / c^{0.87}>1, \ell$ can be comparable to $L$ or even larger. It is an interesting issue whether, in analogy to the pure case, near bulk criticality the presence of the length scale $\ell$, which competes with $L$, has important consequences for the critical Casimir force. In other words, using the following representation of Eq. (4),

$$
f_{\mathrm{C}}(T, L, h) \simeq L^{-d} \hat{\vartheta}\left(L / \xi_{b}, L / \ell\right)
$$

where $\hat{\vartheta}=\vartheta\left(\left(L / \xi_{b}\right)^{1 / \nu},(L / \ell)^{1 /\left(y_{1}-(d-1) / 2\right)}\right)$, we pose the question whether for $L \lesssim \ell$ one can observe significant deviations of the force scaling function $\hat{\vartheta}$ from its universal ordinaryordinary $(o, o)$ fixed-point $(c=\infty, h=0)$ behavior $\hat{\vartheta}\left(L / \xi_{b}, L / \ell=\infty\right)$. We address this question in the following section by using MC simulations.

\section{MONTE CARLO SIMULATIONS}

\section{A. The model and the method}

We have performed MC simulations of an Ising model on a cubic lattice of size $L_{x} \times L_{y} \times L_{z}$ with $L_{x}=L_{y}=6 L_{z}$. Here and in the following all lengths are measured in units of the lattice constant $a_{0}$. The spins $s_{i_{x}, i_{y}, i_{z}}= \pm 1$ are located at every lattice site with the coordinates $i=\left(i_{x}, i_{y}, i_{z}\right), 1 \leq i_{x} \leq L_{x}, 1 \leq i_{y} \leq L_{y}, 1 \leq i_{z} \leq L_{z}$. The Hamiltonian of this lattice model is given by

$$
\mathcal{H}=-J\left(\sum_{\langle n n\rangle} s_{i} s_{i^{\prime}}+\sum_{i_{x}, i_{y}} H_{1}\left(i_{x}, i_{y}\right) s_{i_{x}, i_{y}, 1}+\sum_{i_{x}, i_{y}} H_{2}\left(i_{x}, i_{y}\right) s_{i_{x}, i_{y}, L_{z}}\right),
$$


where $J>0$ is the spin coupling constant, the sum $\langle n n\rangle$ is taken over nearest neighbors, and $H_{1}\left(i_{x}, i_{y}\right)$ and $H_{2}\left(i_{x}, i_{y}\right)$ are dimensionless random fields acting on the top and the bottom surface, respectively, of the system. The surface fields are independent random variables with a Gaussian distribution, with vanishing mean values $\left\langle H_{1}\left(i_{x}, i_{y}\right)\right\rangle=\left\langle H_{2}\left(i_{x}, i_{y}\right)\right\rangle=0$, and with half-widths $h^{2}=\left\langle\left(H_{1}\left(i_{x}, i_{y}\right)\right)^{2}\right\rangle=\left\langle\left(H_{2}\left(i_{x}, i_{y}\right)\right)^{2}\right\rangle$. The computations have been performed for systems with thicknesses $L_{z}=10,15$, and 20. We have used the so-called coupling parameter method in order to determine the $\operatorname{CCF} f_{\mathrm{C}}(\beta, L, h)$, where $\beta=J /\left(k_{B} T\right)$ is the reduced inverse temperature and $L=L_{z}-\frac{1}{2}=9.5,14.5$, and 19.5 is the slab thickness (in units of the lattice spacing) corresponding to the force $f_{\mathrm{C}}(\beta, L, h)$. This method has been employed in previous MC simulations determining the CCF for pure films [14, 19]. We have used the following numerical properties of this Ising model: $\beta_{c}=0.2216544(3)$ [44], $\nu=0.6301(2)$ [36], and $\xi_{0}^{+}=0.501(2)$ [45] in units of the lattice spacing $a_{0}$.

Since according to Eq. (7) the coupling constant within the surface layers and between the surface layers and their neighboring layers is the same as in the bulk, the corresponding surface enhancement is, within mean-field theory and in units of the lattice spacing, $c=1$ [2]. Beyond mean-field theory, the relation between $c$ and the coupling constants is not known. In order to proceed, in the following we set $c^{0.87}=1$ and use the scaling variable $\hat{w}=h / L^{0.26}$. Accordingly, $\ell=h^{3.85}$ so that for the thicknesses $L=9.5,14.5$, and 19.5 used here the condition $\ell \simeq L$ is satisfied for $h=1.80,2.00$, and 2.16, respectively. The value $\hat{w}=0$ corresponds to films with free $(o, o)$ BC.

For every value of the scaling variable $\hat{w}$ we have performed an ensemble average over $N_{r}=64$ independent realizations $j=1,2,3, \ldots, N_{r}$ of random surface fields. For every realization of systems with $L_{z}=10,15,20$ lattice layers the thermal average is performed over $10^{5}, 5 \times 10^{4}$, and $2.5 \times 10^{4}$ hybrid MC steps, respectively, split into 10 series in order to determine the statistical error. We denote $f_{\mathrm{C}}^{j}(\beta, L, h)$ as the critical Casimir force per $k_{B} T_{c}$ and per surface area $S_{2}=L_{x} \times L_{y}$, obtained from the $j$-th realization at the inverse temperature $\beta=1 /\left(k_{B} T\right)$, for the system thickness $L$ and for the random surface field scaling variable $\hat{w}=h / L^{0.26}$. The actual force is computed as an average over all realizations: $f_{\mathrm{C}}(\beta, L, h)=\frac{1}{N_{r}} \sum_{j=1}^{N_{r}} f_{\mathrm{C}}^{j}(\beta, L, h)$. We shall investigate $f_{\mathrm{C}}(\beta, L, h)$ as a function of the two scaling variables $x=\left(L / \xi_{0}^{+}\right)^{1 / \nu} t$ and $\hat{w}=h / L^{0.26}$ (see Eq. (4)):

$$
f_{\mathrm{C}}(\beta, L, h) \simeq L^{-3} \vartheta\left(x=\left(L / \xi_{0}^{+}\right)^{1 / \nu} t, \hat{w}\right)
$$


For the pure system, i.e., $h=0$ we have also obtained $N_{r}$ statistically independent values of the force $f_{\mathrm{C}}^{j}(\beta, L, h=0)$. After averaging over the random surface fields for fixed values of $L$ and of the inverse temperature $\beta$, we obtain the difference $\Delta f$ between the force corresponding to the random surface field $h$ and the corresponding force for a pure system (with $(o, o) \mathrm{BC})$ :

$$
\begin{aligned}
\Delta f(\beta, L, h) & =\frac{1}{N_{r}} \sum_{j=1}^{N_{r}}\left[f_{\mathrm{C}}^{j}(\beta, L, h)-f_{\mathrm{C}}^{j}(\beta, L, h=0)\right] \\
& \simeq L^{-3}[\vartheta(x, \hat{w})-\vartheta(x, \hat{w}=0)] .
\end{aligned}
$$

The statistical error is inferred from 10 series of MC steps for every realization. The variance of different realizations of the disorder field is slightly smaller than the statistical error of a given realization. The error bars shown take into account only the statistical error.

\section{B. Numerical results}

First we check whether, similar to the pure case with nonzero surface fields $H_{1}$, there is a nontrivial dependence of the critical Casimir amplitude on the strength $h$ of the disorder. As mentioned above, for symmetric films the (negative) critical Casimir amplitude as a function of the non-random surface field $H_{1}$ varies from its value at $H_{1}=0$ (ordinary transition fixed point) to its value at $H_{1}=\infty$ (normal transition fixed point) in a non-monotonous way, i.e., through a maximum located at $L \simeq \ell_{\text {ord }}[19,41]$. (In $d=3$, the absolute value of the critical Casimir amplitude for $(o, o) \mathrm{BC}$ is smaller than the one for $(+,+) \mathrm{BC}$, whereas in $d=2$ they are equal.)

In the case of disorder, at $h=0$ the critical Casimir amplitude is the one for $(o, o) \mathrm{BC}$. In analyzing our data for nonzero values of $h$ we have observed that at the bulk critical point the difference $\Delta f$ is vanishingly small. On the other hand, below $T_{c}$ (around $x=$ $\left.\left(L / \xi_{0}^{+}\right)^{1 / \nu} t \simeq-7\right)$ it exhibits a pronounced minimum. Therefore, instead of considering the dependence on $h$ of the critical Casimir amplitude we have studied the critical Casimir force difference $\Delta f$ as a function of $h$ for several fixed values of the temperature scaling variable around the minimum, i.e., $x \approx-2.99,-5.99,-8.98,-11.98$. We have considered $h \in[0,5]$ and have found that, upon increasing $h,|\Delta f|$ increases monotonically with $h$ from 0 to a certain $x$-dependent saturation value at large $h$. Such a leveling off is expected to occur, as discussed in Sec. III In contrast, in the pure case, the small absolute value of 


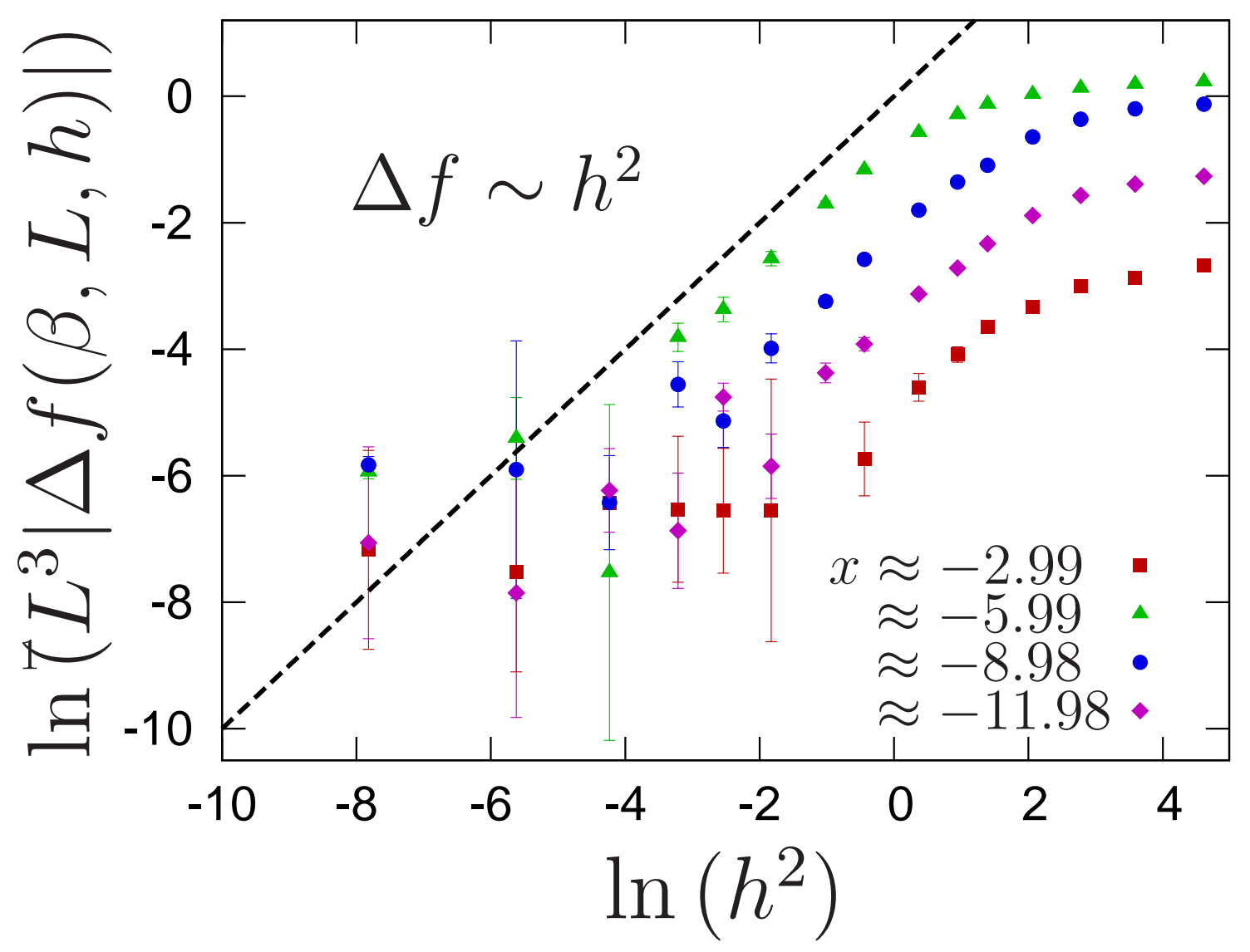

FIG. 1. (Color) Log-log plot of the absolute value of the rescaled force difference $L^{3}|\Delta f(\beta, L, h)|$ as function of $h^{2}$ (Eq. (9) ) for several values of the temperature scaling variable $x=\left(L / \xi_{0}^{+}\right)^{1 / \nu} t \approx$ $-2.99,-5.99,-8.98,-11.98$ and for the system size $L_{z}=L+\frac{1}{2}=15$. The straight dashed line indicates the slope corresponding to the proportionality $\Delta f \sim h^{2}$. The amplitude $\Delta f / h^{2}$ varies as function of $x$.

the Casimir amplitude $\left|\Delta_{(o, o)}\right|$ at first decreases even further upon increasing $H_{1}$ from zero, reaches a minimum, and only then increases towards the large value $\left|\Delta_{(+,+)}\right|$for $(+,+) \mathrm{BC}$ [19, 41]. For small values of $h, \Delta f$ can be described well by a quadratic function of $h$. A crossover from the quadratic dependence to saturation of $\Delta f$ as a function of $h$ occurs above $\ln h^{2} \approx-0.5$ (i.e., $h \approx 0.78$ ), corresponding to $\ell \approx 0.38$ so that $L / \ell \approx 38$. The leveling off occurs for $h \approx 5$, corresponding to $\ell \gg L$ (see Fig. 1).

As the next step we determine the scaling function $\vartheta(x, \hat{w})$. Due to the finite and rather limited sizes of the lattices, which can be studied via MC simulations with presently available resources, one cannot expect to reach the asymptotic regime where the true finite-size scaling 
holds. In order to obtain data collapse and thus being able to infer the leading universal scaling functions, one has to apply corrections both to the scaling function and to the scaling variables. These corrections to scaling are nonuniversal; they depend on details of the model as well as on the geometry and on the boundary conditions [46, 47]. Besides the bulk corrections to scaling, also surface and finite-size ones can occur [48]. Three-dimensional slabs of thickness $L$ exhibit a phase transition of two-dimensional character at a shifted critical point $T_{c}(L)$ with $T_{c}(L \rightarrow \infty)-T_{c}(\infty) \sim L^{-1 / \nu}$. For temperatures near $T_{c}(L)$ one faces considerable finite-size corrections due to the finite lateral system size $L_{x}=L_{y}=L_{\|}$. This leads to a dependence of the critical Casimir forces on the aspect ratio $\rho=L_{z} / L_{\|}$. In the case of periodic BC in the normal direction this dependence is strong for $\rho>1 / 2[49]$. Here, we focus on the film geometry (i.e., $\rho \rightarrow 0$ ), which can be realized in fluid systems by, e.g., wetting films. As it follows from our previous studies [14], for small $\rho$ the scaling function of the critical Casimir force with $(o, o) \mathrm{BC}$ does depend on the aspect ratio but only within a certain interval near its minimum. Here we take $\rho=1 / 6$ and neglect the aspect ratio corrections.

On the other hand, we incorporate those corrections to scaling, which are due to the finite size $L$ in normal direction. In the present study, the following quantities are expected to acquire corrections to scaling:

- the amplitude of the scaling function $\vartheta=L^{3} f_{C}$

- the random surface field scaling variable $\hat{w}$

- the temperature scaling variable $x=\left(L / \xi_{0}^{+}\right)^{1 / \nu} t$.

One may expect that the scaling function of the critical Casimir force $f_{C}$ additionally depends on $L^{-\omega^{\prime}}: f_{C}(\beta, L, h)=L^{-3} \theta\left(x, \hat{w}, L^{-\omega^{\prime}}\right) \simeq L^{-3} \vartheta(x, \hat{w})\left[1+L^{-\omega^{\prime}} \zeta(x, \hat{w})+\ldots\right]$ for $L \gg 1$. (Recall that actually $f_{C}$ depends also on the lattice spacing $a_{0}$ so that the correction to scaling scales as $\left(L / a_{0}\right)^{-\omega^{\prime}}$; we set $a_{0}=1$.) The exponent $\omega^{\prime}$ controls the leading correction to the scaling behavior of the lattice estimate $f_{C}$. In the presence of boundaries, two main corrections to scaling are expected to occur. One is due to the irrelevant bulk scaling fields [50], which introduce exponents $\omega_{i}$ which cannot be expressed in terms of the usual critical exponents such as $y_{t}$ and $y_{b}$. The latest estimate for the value of the smallest, and thus most relevant, of those exponents is $\omega \approx 0.832(6)$ for the $d=3$ Ising model [51]. The other 
correction terms stem from the irrelevant surface contribution to the Hamiltonian $\mathcal{H}$ [2]. The value of $\omega^{\prime}$ is determined by that irrelevant surface or bulk scaling field which has the smallest scaling dimension and which also affects $f_{C}$. The influence of the bulk corrections to scaling can be reduced by using improved Hamiltonians and observables, which can also serve as representatives of the same universality class. This is described in detail in Ref. [36]. The value of the exponent, corresponding to the least irrelevant surface contributions in our system, is not known. Even if the bulk correction-to-scaling exponent is dominant, by fitting the data it is difficult to disentangle corrections $\propto L^{-\omega}$ and $\propto L^{-1}$. The latter can occur due to the presence of the boundaries. Moreover, for small lattice sizes, next-to-leading corrections to scaling might also be numerically important, resulting in effective exponents. The current accuracy of our MC data and the range of sizes $L$ investigated here do not allow for the reliable determination of $\omega^{\prime}$, the function $\zeta(x, \hat{w})$, and the effective exponents. The analysis of various observables [15] revealed that also $x$ acquires a leading Wegner correction [50] of the form $x \equiv \tau\left(L / \xi_{0}^{+}\right)^{1 / \nu}\left(1+g_{\omega} L^{-\omega}\right)$. A detailed analysis of all types of corrections is beyond the scope of the present study and is left to future research.

In our previous MC simulations aimed at obtaining critical Casimir forces for Ising films with a variety of universal boundary conditions [2], such as $(+,+),(+,-)$, or $(o, o) \mathrm{BC}$ [14, 19], corrections to scaling were taken into account in an effective way by using various ansätze. The choice for a particular form of corrections to scaling was guided by achieving the best data collapse or the best fits. With the lack of corresponding theoretical guidance, in the present study we have adopted the same, pragmatic approach. First, as a phenomenological ansatz for the effective corrections we take $\omega^{\prime} \simeq \omega \simeq 1$. Second, we follow a well established procedure of incorporating corrections to scaling by introducing an effective thickness $L+\hat{\delta}$ [16, 19, 37, 52]. Accordingly, our ansatz for the corrections to scaling is

$$
f_{C}(\beta, L, h)=(L+\hat{\delta}(h))^{-3} \vartheta\left(\left[(L+\hat{\delta}(h)) / \xi_{0}^{+}\right]^{1 / \nu} t,(L+\hat{\delta}(h))^{-1 /\left(y_{1}-(d-1) / 2\right)} h\right) .
$$

(We note that this way the leading bulk correction to scaling is treated "effectively", because the correction of the the scaling function $(L+\hat{\delta}(h))^{-3}$ has the expansion $L^{-3}(1-3 \hat{\delta}(h) / L+$ ....).) The nonuniversal length $\hat{\delta}(h)$ is fixed in such a way that the data scatter due to different $L$ is minimal. In order to employ such a correction-to-scaling scheme the knowledge of the whole surface $\vartheta(x, \hat{w})$ is needed, which is computationally too demanding (see the Appendix in Ref. [14(b)] where we have discussed in detail our strategy of obtaining the best fit for 
the values of the parameters which control the corrections to scaling). In our simulations we have generated data which correspond to only several cuts of the surface $\vartheta(x, \hat{w})$ along $\hat{w}=$ const. Therefore we apply corrections to scaling along these cuts by introducing $\delta(\hat{w})$ and the effective thickness $L_{\text {eff }}(\hat{w})=L+\delta(\hat{w})=L_{z}-0.5+\delta(\hat{w})$. Accordingly, we introduce a corrected scaling variable

$$
y=y(x, \hat{w}, L)=\left(\frac{L_{\mathrm{eff}}(\hat{w})}{\xi_{0}^{+}}\right)^{1 / \nu} t=\left(\frac{L_{\mathrm{eff}}(\hat{w})}{L}\right)^{1 / \nu} x=\left(1+\frac{\delta(\hat{w})}{L}\right)^{1 / \nu} x .
$$

Plotting $L_{\text {eff }}^{3} f_{C}(\beta, L, h)$ versus $y$ for fixed $\hat{w}$ and choosing $\delta(\hat{w})$ such that data collapse is promoted for large $L$, one obtains a scaling function $g(y, \hat{w})=L_{\text {eff }}^{3} f_{C}(\beta, L, h)$, which for large $L$ does not exhibit an explicit dependence on $L$. (This is achieved for smaller values of $L$ than for the scaling leading to the scaling function $\vartheta(x, \hat{w})$ introduced before by considering $L^{3} f_{C}(\beta, L, h)$.) From the knowledge of the scaling function $g(y, \hat{w})$ one can construct the desired scaling function $\vartheta(x, \hat{w})$ according to $\vartheta(x, \hat{w})=g(y=x, \hat{w})+O(\delta(\hat{w}) / L)$.

The nonuniversal parameters $\delta(\hat{w})$ are fixed in such a way that the data collapse of the Monte Carlo data for $L=10,15$, and 20 is optimal in the region $-10<y<-2$. Our corresponding results for $\delta(\hat{w})$ are presented in Table I.

TABLE I. Correction-to-scaling parameter $\delta(\hat{w})$ (see Eq. (11))

\begin{tabular}{|c||c|c|c|c|c|c|}
\hline$\hat{w}$ & 0 & 0.25 & 0.5 & 0.75 & 1 & 2 \\
\hline$\delta(\hat{w})$ & $1.6(1)$ & $1.7(1)$ & $1.67(10)$ & $1.4(1)$ & $1.05(10)$ & $0.1(1)$ \\
\hline
\end{tabular}

The results of the above procedure depend on the range of $y$ considered for the data collapse.

By applying the rescaling procedure as described above, we have obtained an estimate for the scaling function $\vartheta(x, \hat{w})$ of the Casimir force $f_{C}(\beta, L, h)$ for the $3 d$ Ising model in the slab geometry with random surface fields. It is shown in Fig. 2 as a function of the scaling variable $x$ for the values $\hat{w}=0,0.25,0.5,0.75,1$, and 2 . One sees that the rescaling procedure leads to data collapse for various system sizes $L$, for a given value of $\hat{w}$. However, the rescaled data for different values of $\hat{w}=0,0.25,0.5$ do not collapse. The curves for $\hat{w}=0.75,1$ and 2 lie almost on the top of each other. Note that $\hat{w}=0.75$ and 1 lie in the crossover regime to the "strong disorder limit", where $L<\ell$, whereas for $\hat{w}=2$ this limit is 


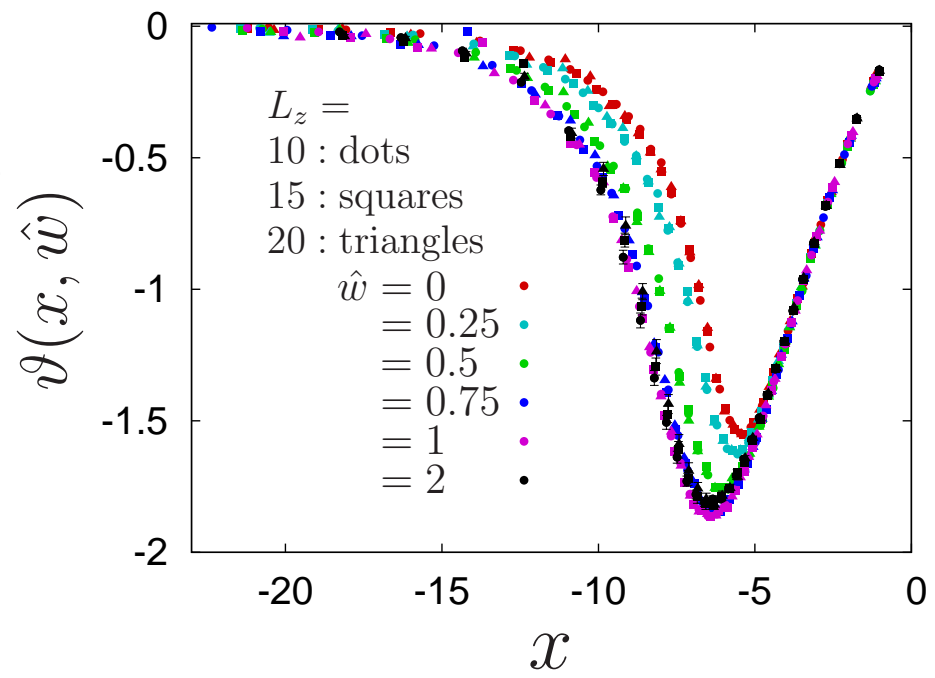

FIG. 2. (Color) Scaling function $\vartheta(x, \hat{w})($ Eq. (8) $)$ of the critical Casimir force for $3 d$ Ising slabs with random surface fields for several values of the random surface field scaling variable $\hat{w}=0,0.25,0.5,0.75,1$ and 2 (from top to bottom). This scaling function has been obtained according to the procedure described in the main text. The MC data reported in this figure refer to slabs with thicknesses $L_{z}=10$ (dots), 15 (squares), 20 (triangles) and indicate that for fixed $\hat{w}$ data collapse has been accomplished. For $\hat{w} \leq 0.75$ the error bars are smaller than the symbol size. The pure case $\hat{w}=0$ is also shown (see Ref. [14]).

almost attained (compare Fig. 1). For strong disorder, as discussed earlier, the majority of the surface spins do not fluctuate but are frozen to the values -1 or +1 so that as a function of $h^{2}$ the contribution $\Delta f$ to the Casimir scaling function due to the random surface fields levels off, i.e., in this limit the value of $h$ does not matter.

In order to gain more insight into the effect of random surface fields on our system, we use the estimate for $\vartheta(x, \hat{w})$ shown in Fig. 2 and calculate for each value of $\hat{w}$ the difference $\Delta f$ according to Eq. (91). This is done by subtracting from the curve corresponding to the particular value of $\hat{w}$ the one corresponding to the pure case of $\hat{w}=0$ (red curve). In Fig. 3(a) we show the result of this operation rescaled by $L_{\text {eff }}^{3}$ for an Ising slab with $L_{z}=15$ and for a random surface field $h=1.01(8)$ corresponding to the scaling variable $\hat{w}=0.5$ for three cases: (i) random surface fields applied only on the top side of the film (t), (ii) only on the bottom side (b), and (iii) on both sides ( $\&$ b). Obviously the results for the (t) and (b) cases coincide. For comparison we have plotted also the results for the bottom 

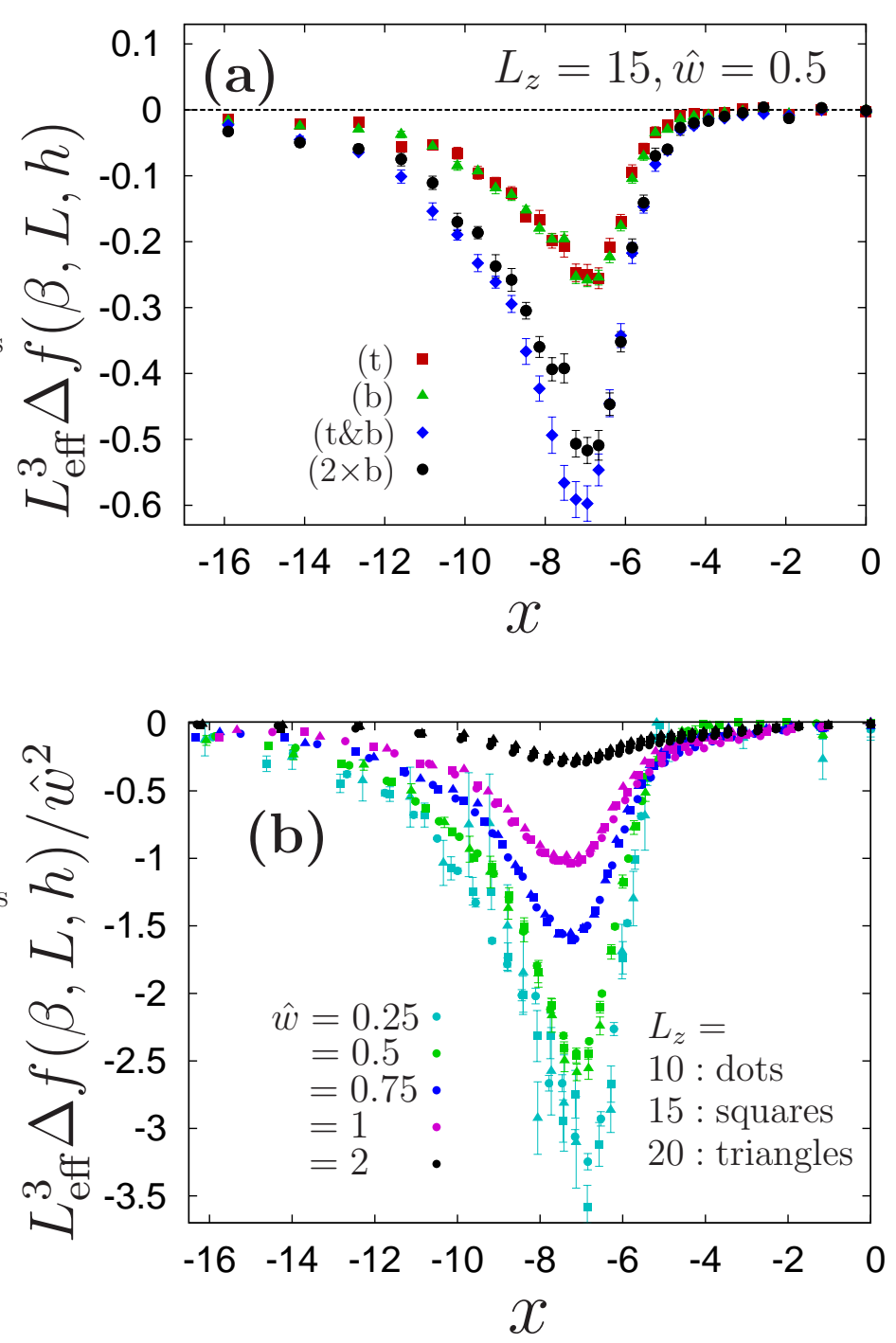

FIG. 3. (Color) (a) Additivity check: rescaled difference $L_{\mathrm{eff}}^{3} \Delta f(\beta, L, h)$ as function of the scaling variable $x$ (see Eq. (9) and main text) for the system size $L_{z}=15$ and $h=1.01(8)$ corresponsing to $\hat{w}=0.5$. Random surface fields present only at the top (t), only at the bottom (b) (which has to yield identical results), and on both sides of the system ( $\&$ b). The data for bottom random fields multiplied by 2 are shown for comparison (note that $2 \times b \equiv(t)+(b)$ ). The difference between the black and blue data highlights the lack of additivity which leads to stronger forces. In all plots $\hat{w}=h / L^{0.26}$. (b) Rescaled difference, between the case of the presence and of the absence of randomness, of the Casimir force scaling function $L_{\text {eff }}^{3} \Delta f(\beta, L, h) / \hat{w}^{2}$ of the $3 d$ Ising model in the slab geometry as a function of the scaling variable $x$. The data correspond to the same thicknesses $L_{z}$ as in Fig. 2, The curves correspond to $\hat{w}=0.25,0.5,0.75,1,2$ (from bottom to top). For $\hat{w}>0.75$ the error bars are smaller than the symbol size. 
side multiplied by two $(2 \times b \equiv(t)+(b))$. If the effects from the top and the bottom sides were additive, $(\mathrm{t} \& \mathrm{~b})$ should coincide with $(2 \times \mathrm{b})$, which is not the case; the actual force is stronger. Thus we conclude that the contribution to the CCF stemming from the random fields at both confining surfaces is not the sum of the contributions due to the random surface fields being present only at one of the two surfaces.

Finally, in order to focus on the leading behavior of the difference $\Delta f$ between the Casimir scaling functions for a system with and without disorder, in Fig. 3(b) we plot $L_{\text {eff }}^{3} \Delta f(\beta, L, h) / \hat{w}^{2}$ for various values of $L_{z}$ and $\hat{w}$ as function of the scaling variable $x$. For $\hat{w}=0.25$ and 0.5 , we observe good data collapse within the error bars of our data, confirming that as function of $\hat{w}$ the leading behavior of the difference $\Delta f$ for small $\hat{w}$ is quadratic in $\hat{w}$, consistent with the results shown in Fig. 1. Based on these observations and strengthened by the symmetry property $p\left(H_{1}\right)=p\left(-H_{1}\right)$ of the surface field distribution, we put forward the hypothesis that for small $\hat{w}$ one has

$$
f_{\mathrm{C}}(\beta, L, h) \simeq L^{-3}\left\{\vartheta\left(\left(L / \xi_{0}^{+}\right)^{1 / \nu} t, \hat{w}=0\right)+\hat{w}^{2} \vartheta_{0}\left(\left(L / \xi_{0}^{+}\right)^{1 / \nu} t\right)\right\}
$$

where $\vartheta\left(\left(L / \xi_{0}^{+}\right)^{1 / \nu} t, \hat{w}=0\right)$ is the scaling function of the critical Casimir force for $(o, o)$ BC. The universal scaling function $\vartheta_{0}(x)$ depends on $x$ only; $\vartheta_{0}(x)$ is given by the curve in Fig. 33(b) formed by the data corresponding to $\hat{w}=0.25$ and 0.5 . We note that Eq. (12) agrees with the expansion in Eq. (5) with a vanishing leading correction-to-scaling term. From this we infer that the randomness induced occurrence of the extra contribution $\Delta f$ to the critical Casimir force is due to the irrelevant scaling field $h$ (or more generally $\tilde{g}_{1}=h / c^{y_{c}}$ ). Moreover, at large $h$ such that $\ell \geq L$ we observe surface spin configurations of randomly distributed frozen spins (see the discussion ath the end of Sec. II). For the corresponding CCF one has

$$
f_{\mathrm{C}}(\beta, L, h) \simeq L^{-3} \vartheta\left(\left(L / \xi_{0}^{+}\right)^{1 / \nu} t, \hat{w}=\infty\right)=L^{-3} \vartheta_{w}\left(\left(L / \xi_{0}^{+}\right)^{1 / \nu} t\right)
$$

where the scaling function $\vartheta_{w}(x)$ is approximately given by the curve in Fig. 2 which is common to the data points corresponding to $\hat{w}=1$ and 2 . We note that for large $h$ the scaling analysis, which leads to the conclusion that the disorder is an irrelevant perturbation of the ordinary surface universality class, does not hold. Our findings that CCF in films are significantly influenced by the surface disorder for $L / \ell \approx 1$ should actually be valid not only for thin slabs but for all slabs in the scaling limit $L \gg 1$ and $\ell=\left(\tilde{g}_{1}\right)^{-1 /\left(y_{1}-(d-1) / 2\right)} \gg 1$ 
such that $L / \ell$ is kept nonzero and finite, which requires large values of $\tilde{g}_{1}$. The present limits of the accuracy of our data do not allow us to draw quantitatively reliable conclusions concerning the behavior of $\Delta f$ above $T_{c}$.

\section{CONCLUSIONS}

The MC simulation data show that the presence of random surfaces fields with zero mean increases substantially the strength of the critical Casimir force as compared with the pure case without fields. The strongest effects occur when the length scale $\ell$ associated with the random surface field becomes comparable with the thickness of the film. For weak disorder this effect is proportional to the square of the strength of the random surface fields. For strong disorder, the dependence of the CCF on $h$ levels off. For all strengths of disorder, at bulk criticality the CCF decays asymptotically as function of the film thickness $L$ as $L^{-3}$, which is the same behavior as for the pure system.

As mentioned in the Introduction, in the study of Ref. [30] the quenched random disorder is applied only to one of the two surfaces. Moreover, different from the present work, it is governed by the binomial distribution, i.e., spins on the surface subjected to disorder take the value 1 with the probability $p$ and -1 with the probability $1-p$. On the other surface the spins are fixed at the value +1 or -1 . For $p=0.5$, for which the mean value of the surface fields vanishes, the Monte Carlo simulation data for CCFs presented in Ref. [30] scale with the inverse third power of the (effective) film thickness. This is interesting, because the binomial distribution used in Ref. [30] represents a sort of "strong disorder" limit; nevertheless, the critical behavior is still governed by the ordinary fixed point. This is consistent with our findings. For other values of $p$ considered in Ref. [30] a substantial dependence of the scaling function on $L$ is observed. As suggested by the author, this latter lack of data collapse may be due to the negligence of the scaling variable associated with the random surface field describing the crossover from the ordinary to the normal phase transition. In our case, taking into account the scaling variable connected with the random surface field $h$ was necessary in order to achieve data collapse.

Our theoretical predictions lend themselves to be investigated experimentally and pose a challenge to further analytic studies. The model studied here can be realized experimentally by confining a near-critical binary mixture such as lutidine-water by two planar walls, 
each of them patterned by chemical stripes with alternating, strong preferences for the two species of the binary liquid mixture. In the limit of narrow stripes these surfaces mimic the ordinary surface universality class [22]. The random surface fields can be realized by randomly adsorbing on these surface structures a binary mixture of adsorbates with opposite preferences for the two liquid species. The critical Casimir force can be obtained by AFM where the two surfaces are those of two crossed cylinders with large radii of curvature [53].

[1] M. E. Fisher and P. G. de Gennes, C. R. Acad. Sci. Paris Ser. B 287, 207 (1978).

[2] K. Binder in Phase Transitions and Critical Phenomena, edited by C. Domb and J. L. Lebowitz (Academic, New York, 1983), Vol. 8, p. 1; H. W. Diehl, ibid. Vol. 10 (1986) p. 76.

[3] R. Garcia and M. H. W. Chan, Phys. Rev. Lett. 83, 1187 (1999); A. Ganshin, S. Scheidemantel, R. Garcia, and M. H.W. Chan, ibid. 97, 075301 (2006).

[4] M. Fukuto, Y. F. Yano, and P. S. Pershan, Phys. Rev. Lett. 94, 135702 (2005).

[5] S. Rafaï S., D. Bonn, and J. Meunier, Physica A 386, 31 (2007).

[6] C. Hertlein, L. Helden, A. Gambassi, S. Dietrich, and C. Bechinger, Nature 451, 172 (2008).

[7] A. Gambassi, A. Maciołek, C. Hertlein, U. Nellen, L. Helden, C. Bechinger, and S. Dietrich, Phys. Rev. E 80, 061143 (2009).

[8] U. Nellen, J. Dietrich, L. Helden, S. Chodankar, K. Nygard, J. F. van der Veen, and C. Bechinger, Soft Matter 7, 5360 (2011).

[9] R. Garcia and M. H. W. Chan, Phys. Rev. Lett. 88, 086101 (2002).

[10] M. Krech and S. Dietrich, Phys. Rev. A 46, 1886 (1992); ibid. 46, 1922 (1992).

[11] M. Krech, Phys. Rev. E 56, 1642 (1997).

[12] Z. Borjan and P. J. Upton, Phys. Rev. Lett. 81, 4911 (1998); ibid. 101, 125702 (2008).

[13] A. Maciołek and S. Dietrich, Europhys. Lett. 74, 22 (2006).

[14] (a) O. Vasilyev, A. Gambassi, A. Maciołek, and S. Dietrich, EPL 80, 6000 (2007); (b) Phys. Rev. E 79, 041142 (2009).

[15] A. Hucht, Phys. Rev. Lett. 99, 185301 (2007).

[16] M. Hasenbusch, Phys. Rev. B 82, 104425 (2010).

[17] T. F. Mohry, A. Maciołek, and S. Dietrich, Phys. Rev. E 81, 061117 (2010).

[18] D. B. Abraham and A. Maciołek, Phys. Rev. Lett. 105, 055701 (2010). 
[19] O. Vasilyev, A. Maciołek, and S. Dietrich, Phys. Rev. E 84, 041605 (2011).

[20] F. M. Schmidt and H. W. Diehl, Phys. Rev. Lett. 101, 100601 (2008).

[21] U. Nellen, L. Helden, and C. Bechinger, EPL 88, 26001 (2009).

[22] M. Tröndle, O. Zvyagolskaya, A. Gambassi, D. Vogt, L. Harnau, C. Bechinger, and S. Dietrich, Mol. Phys. 109, 1169 (2011).

[23] A. Naji, D. S. Dean, J. Sarabadani, R. R. Horgan, and R. Podgornik, Mol. Phys.109, 1169 (2011).

[24] D. Ben-Yaakov, D. Andelman, and H. Diamant, Phys. Rev. E 87, 022402 (2013).

[25] S. Dietrich in Phase Transitions and Critical Phenomena edited by C. Domb and J. L. Lebowitz, Vol. 12 (Academic, London, 1988), p.1; G. Forgacs, R. Lipowsky, and T. M. Nieuwenhuizen, ibid. Vol. 14 (1991), p. 135.

[26] K. K. Mon and M. P. Nightingale, Phys. Rev. B 37, 3815 (1988).

[27] H. W. Diehl and A. Nüsser, Z. Phys. B 79, 69 (1990).

[28] J. L. Cardy, J. Phys. A: Math. Gen. 24, L1315 (1991).

[29] M. Pleimling, J. Phys. A: Math. Gen. 37, R79 (2004).

[30] F. Parisen Toldin, arXiv:1308.5220v1 (2013).

[31] M. Blume, Phys. Rev. 141, 517 (1966).

[32] H. W. Capel, Physica 32, 966 (1966).

[33] F. Karimi Pour Haddadan, A. Naji, A. Khame Seifi, and R. Podgornik, J. Phys.: Condensed Matt. 26, 075103 (2014).

[34] R. Guida and J. Zinn Justin, J. Phys. A: Math. Gen. 31, 8103 (1998).

[35] D. Andelman and A. N. Berker, Phys. Rev. B 29, 2630 (1984).

[36] A. Pelissetto and E. Vicari, Phys. Rep. 368, 549 (2002).

[37] M. Hasenbusch, Phys. Rev. B 84, 134405 (2011); ibid. 83, 134425 (2011).

[38] H. W. Diehl, Int. J. Mod. Phys. B 11, 3503 (1997).

[39] U. Ritschel and P. Czerner, Phys. Rev. Lett. 77, 3645 (1996).

[40] A. Ciach and U. Ritschel, Nucl. Phys. B 489, 653 (1997).

[41] A. Maciołek, A. Ciach, and A. Drzewiński, Phys. Rev. E 60, 2887 (1999).

[42] A. Maciołek, R. Evans, and N. B. Wilding, J. Chem. Phys. 119, 8663 (2003).

[43] A. Maciołek, A. Drzewiński, and P. Bryk, J. Chem. Phys. 120, 1925 (2004).

[44] A.L. Talapov and W.J. Blöte, J. Phys. A: Math. Gen. 29, 5727 (1996). 
[45] C. Ruge, P. Zhu, and F. Wagner, Physica A 209, 431 (1994).

[46] V. Privman, and M. E. Fisher, J. Phys. A 16, L295 (1983).

[47] J. M. Luck, Phys. Rev. B 31, 3069 (1985).

[48] V. Privman, in Finite Size Scaling and Numerical Simulations of Statistical Systems, edited by V. Privman (World Scientific, Singapore, 1990), p. 1.

[49] A. Hucht, D. Grüneberg, and F. M. Schmidt, Phys. Rev. E 83, 051101 (2011).

[50] F. J. Wegner, Phys. Rev. B 5, 4529 (1972).

[51] M. Hasenbusch, Phys. Rev. B 82, 174433 (2010).

[52] M. Hasenbusch, Phys. Rev. B 85, 174421 (2012).

[53] J. Israelachvili, Intermolecular and Surface Forces. 2nd ed. (Academic, New York, 1990). 